\title{
Mesenchymal stem cell-derived angiogenin promotes primodial follicle survival and angiogenesis in transplanted human ovarian tissue
}

\author{
Yaoyao Zhang ${ }^{1,2,3}, X_{i} X^{11,4}$, Jie Yan ${ }^{1,2,3}$, Liying Yan ${ }^{1,2,3}$, Cuilin Lu ${ }^{1,2,3}$, Xiaohui Zhu ${ }^{1,2,3}$, Tianren Wang ${ }^{1,2,5}$, \\ Tailang Yin ${ }^{1,2,3}$, Rong $\mathrm{Li}^{1,2,3}$, Hsun-Ming Chang ${ }^{6}$ and Jie Qiao ${ }^{1,2,3^{*}}$
}

\begin{abstract}
Background: We have recently reported that human bone marrow-derived mesenchymal stem cells (MSCs) facilitate angiogenesis and prevent follicle loss in xenografted human ovarian tissues. However, the mechanism underlying this effect remains to be elucidated. Thus, determining the paracrine profiles and identifying the key secreted factors in MSCs co-transplanted with ovarian grafts are essential for the future application of MSCs.

Methods: In this study, we used cytokine microarrays to identify differentially expressed proteins associated with angiogenesis in frozen-thawed ovarian tissues co-transplanted with MSCs. The function of specific secreted factors in MSCs co-transplanted with human ovarian tissues was studied via targeted blockade with short-hairpin RNAi and the use of monoclonal neutralizing antibodies.

Results: Our results showed that angiogenin (ANG) was one of the most robustly up-regulated proteins (among 42 protein we screened, 37 proteins were up-regulated). Notably, the targeted depletion of ANG with short-hairpin RNAi (shANG) or the addition of anti-ANG monoclonal neutralizing antibodies (ANG Ab) significantly reversed the MSC-stimulated angiogenesis, increased follicle numbers and protective effect on follicle apoptosis.

Conclusion: Our results indicate that ANG plays a critical role in regulating angiogenesis and follicle survival in xenografted human ovarian tissues. Our findings provide important insights into the molecular mechanism by which MSCs promote angiogenesis and follicle survival in transplanted ovarian tissues, thus providing a theoretical basis for their further application.
\end{abstract}

Keywords: Ovarian tissue transplantation, Mesenchymal stem cell, Follicle survival, Fertility preservation, Angiogenin

\section{Background}

The transplantation of frozen-thawed ovarian tissue is a promising technique for the restoration of endocrine function and fertility [1], especially in cancer patients who have undergone gonadotoxic therapy [2-6]. However, ischemia-reperfusion injury and insufficient revascularization following transplantation, which lead to

\footnotetext{
* Correspondence: jie.qiao@263.net

'Department of Obstetrics and Gynecology, Center for Reproductive Medicine, Peking University Third Hospital, No.49 North HuaYuan Road, HaiDian District, Beijing 100191, China

${ }^{2}$ Beijing Key Laboratory of Reproductive Endocrinology and Assisted Reproduction, Beijing 100191, China

Full list of author information is available at the end of the article
}

failure of follicular survival, are significant obstacles to the wider application of this technique [7-9]. Therefore, searching for methods to minimize this damage and to induce adequate revascularization is a critical step for successful ovarian tissue transplantation and the preservation of fertility.

Mesenchymal stem cells (MSCs), which can be isolated from various tissues [10], are a population of cells possessing pluripotent capabilities. MSCs have been shown to be a promising therapeutic approach to treat a spectrum of diseases, especially in disorders associated with insufficient angiogenesis $[11,12]$. Recently, we demonstrated that MSCs from human bone marrow 
functioned as a supportive agent for human ovarian tissue transplantation, promoting angiogenesis and follicle survival [13]. However, the mechanism underlying this process is unknown. A better understanding of how co-transplanted MSCs exert their pro-angiogenic effects on ovarian grafts can help optimize the parameters for clinical applications. Therefore, in this study, we sought to investigate the possible mechanisms by which human MSCs promote re-vascularization and prevent follicle loss in xenografted ovarian tissues.

Angiogenesis is a multi-step process that involves the survival, proliferation, migration, and differentiation of endothelial cells, as well as tube formation and maturation. In recent years, several studies have used different approaches to investigate the mechanisms by which MSCs facilitate angiogenesis; however, no consistent conclusion has been reached. Some studies have indicated that MSCs are capable of differentiating into endothelial cells, pericytes, or even vessel walls to support the formation of blood vessels [14-16]. Other studies have suggested that MSCs are capable of protecting endothelial cells from apoptosis, including from oxidative stressrelated apoptosis in the initial phase of angiogenesis [17]. Furthermore, in addition to the early steps of angiogenesis, such as endothelial cell proliferation, MSCs have been reported to support the late phases of angiogenesis, including blood vessel maturation $[18,19]$. However, these proposed mechanistic explanations are still debated. Conflicting data have shown that morphological and ultrastructural evidence of MSC differentiation into endothelial cells or blood vessel wall structures in vivo is uncommon. In addition, the integration of the transplanted MSCs into host blood vessels has not been observed in animal studies [20].

In the past several years, research has focused on the secretion-based paracrine regulatory role of MSCs. Studies have shown that MSCs can establish a proangiogenic microenvironment by persistently secreting bioactive molecules that promote angiogenesis and microvascular network formation [21]. Furthermore, accumulating reports have suggested that the therapeutic potential of MSCs is largely dependent on their secretory capacity rather than on their differentiation capacity [22]. Studies of MSC secretion profiles have shown that some pro-angiogenic cytokines, such as VEGF, MCP-1 and FGF-2, can be detected in the conditioned medium of MSCs [23]. However, the composition of the profile of secreted molecules under different conditions varies greatly, leading to differences in angiogenic capacities [24]. For example, it has been reported that under hypoxic conditions, the secretion of proangiogenic factors can be significantly increased [25]. Due to these variations and the limited knowledge regarding the cytokine production of MSCs following transplantation (a hypoxic condition), determining the paracrine profiles and identifying the key secreted cytokines in MSCs co-transplanted with ovarian tissues are essential steps before further (pre)clinical studies can be performed. Therefore, in the present study, we designed an antibody-based cytokine microarray to investigate the expression profiles of angiogenesis-related proteins in frozen-thawed ovarian tissues co-transplanted with MSCs. We found that a panel of proteins, including angiogenin (ANG), was significantly up-regulated. Hence, we sought to suppress ANG secretion in MSCs via RNAi with an ANG-specific short-hairpin RNA (shANG) or using ANG monoclonal antibodies to explore the role of ANG in angiogenesis and follicle survival induced by MSCs in ovarian transplantation.

\section{Methods}

\section{Identification and isolation of human MSCs}

The protocols for the collection of human MSCs and ovarian samples were approved by the Ethics Committee of the Peking University Third Hospital. Informed consent was obtained from all subjects. Human MSCs were isolated by density gradient centrifugation (Additional file 1: Figure S1). The detailed methods were described previously [26]. Human MSCs were isolated from the bone marrow of a healthy female (age: 28 years old) who underwent bone marrow harvesting for allogeneic bone marrow transplantation at our hospital. Human MSCs were cultured in alpha minimum essential Eagle's medium ( $\alpha$-MEM) supplemented with 20\% human serum albumin (Life Technologies, Carlsbad, CA), Lglutamine and penicillin-streptomycin (Invitrogen, Life Technologies, Grand Island, NY) and used for experiments during passages 3 to 8 . For identification, the MSCs were stained with antibodies against CD34, CD44, CD45, CD19, CD90, and CD105 (Biolegend, San Diego, CA). The detail information about surface marker selection and stemness potential characterization of human MSCs were provided previously [26].

\section{Lentivirus production and infection}

An ANG-specific short-hairpin RNAi (shANG) was synthesized and cloned into a lentiviral GV248 vector (Genechem, China). The sequences for RNAi were designed to knock down the gene expression of ANG (shANG) or were control particles (shCTRL) and were obtained using the shRNA library of the RNAi Codex (www.codex.cshl.edu).

The target sequences were as follows: shANG: $5^{\prime}$ - CC ACTTGGATCAGTCAATT -3' and shCTRL: 5'-AAC AGTCGCGTTTGCTACTTT-3'. Lentivirus preparation, infection and selection were performed according to the technical manual for the vector. 


\section{Quantitative real-time PCR and ELISA}

Total RNA was extracted from MSCs transfected with shANG or shCTRL using a Total RNA Purification Plus Kit (Norgen, Canada), according to the manufacturer's instructions. A total of $1.0 \mu \mathrm{g}$ of RNA was reversetranscribed using the SuperScript III First-strand Synthesis System. PCR amplification was carried out in an ABI 7500 Detection System using Power SYBR (Applied Biosystems, Carlsbad, CA) under the following conditions: $50{ }^{\circ} \mathrm{C}$ for $2 \mathrm{~min} ; 95{ }^{\circ} \mathrm{C}$ for $2 \mathrm{~min}$; and 40 cycles of $95{ }^{\circ} \mathrm{C}$ for $15 \mathrm{~s}, 60{ }^{\circ} \mathrm{C}$ for $15 \mathrm{sec}$, and $72{ }^{\circ} \mathrm{C}$ for $1 \mathrm{~min}$. GAPDH was used as a reference gene for normalization. The level of ANG mRNA expression was calculated using the following formula: $2^{(\Delta \mathrm{Ct} \text { Test }-\Delta \mathrm{Ct} \text { Control) }}$. The $C t$ of ANG was compared with that of the internal control GAPDH gene.

The primer sequences used for PCR were as follows:

GAPDH sense: 5' - TGACTTCAACAGCGACACCCA $-3^{\prime}$ and antisense: 5'- CACCCTGTTGCTGTAGCCA AA -3'; ANG sense: 5' - CCTCCATGCCAGTACCGAG $-3^{\prime}$ and antisense: 5' - GGACGACGGAAAATTGAC TGA -3 '.

We used an ELISA kit (R\&D, Abingdon, UK) for the quantitative measurement of human ANG in the conditioned media of MSCs transfected with specific shANG or shCTRL after $24 \mathrm{~h}$ of culture. MSCs were plated on a 6-well plate at a density of $10^{5}$ cells/well. ELISAs were performed according to the manufacturer's instructions. Each sample was analyzed in triplicate.

\section{Collection and treatment of human ovarian tissue}

The use of human ovarian tissues was reviewed and approved by the ethics committee of Peking University (registration number: 2009005). Human ovarian tissue was obtained from a 26-year-old female patient who underwent gender reassignment surgery. One biopsy from each ovary was obtained and cut into small pieces after removing the medulla tissues. The ovarian tissues were cryopreserved and thawed as previously described [26]. Briefly, the ovarian tissue was transported from the operating room to the laboratory in Leibovitz's L-15 medium (Invitrogen, Carlsbad, CA) supplemented with $1 \%$ human serum albumin (Life Technologies, Carlsbad, CA), $100 \mathrm{IU} / \mathrm{mL}$ penicillin (Sigma, St. Louis, MO) and $100 \mu \mathrm{g} / \mathrm{mL}$ streptomycin (Sigma, St. Louis, MO). After enucleating the medulla with surgical scissors and a scalpel, the ovarian cortical tissues were manually cut into small pieces with a size of $5 \mathrm{~mm} \times 5 \mathrm{~mm} \times 1 \mathrm{~mm}$ (thickness).

Two slices of ovarian cortical tissues were placed in a 1.8-mL cryovial (Nunc, Roskilde, Denmark) containing $1 \mathrm{~mL}$ of $1.5 \mathrm{~mol} / \mathrm{L}$ DMSO (Sigma-Aldrich, St. Louis, MO), $0.1 \mathrm{~mol} / \mathrm{L}$ sucrose (Sigma-Aldrich, St. Louis, MO) and 10\% HSA (Life Technologies, Carlsbad, CA) in
Leibovitz medium. After $30 \mathrm{~min}$ of exposure to the cryoprotective agent at $4{ }^{\circ} \mathrm{C}$, the cryovial was transferred to a program freezer (Biomed Freezer Kryo 10, series II; Planer, Middlesex, UK). The freezing and thawing procedures were carried out according to the techniques described by Andersen [27].

\section{Ovarian transplantation in severe combined immune deficiency mice}

All animal procedures were approved by the Institutional Animal Care and Use Committee of the Peking University Third Hospital. A total of 30 8-week-old female ovariectomized mice with severe combined immune deficiency (SCID) (Animal Center of Medical College of Peking University) were used in the study. Consistently with previous reports [13, 28, 29], each ovarian fragment was co-transplanted with $5 \times 10^{5}$ MSCs in our study,.

For the array analysis, 6 mice were randomly assigned to one of 2 groups: (1) Graft + MSC group: each ovarian fragment was transplanted with $5 \times 10^{5}$ MSCs packaged in $10 \mu \mathrm{L}$ of Matrigel (Corning, USA); and (2) Graft group (control): each ovarian fragment was transplanted in $10 \mu \mathrm{L}$ of Matrigel. The grafts were retrieved and rapidly frozen in liquid nitrogen for cytokine array analyses 7 days after transplantation.

For the shRNA blockade experiment, 12 mice were divided into 4 equal groups: (1) Graft group: each ovarian fragment was transplanted with $10 \mu \mathrm{L}$ of Matrigel; (2) Graft + MSC group: each ovarian fragment was transplanted with $5 \times 10^{5}$ MSCs packaged in $10 \mu \mathrm{L}$ of Matrigel; (3) Graft + shCTRL MSC group: each ovarian fragment was transplanted with $5 \times 10^{5}$ shCTRL- transfected MSCs packaged in $10 \mu \mathrm{L}$ of Matrigel; and (4) Graft + shANG MSC group: each ovarian fragment was transplanted with $5 \times 10^{5}$ stably shANG-transfected MSCs packaged in $10 \mu \mathrm{L}$ of Matrigel.

For the antibody blockade experiment, 12 mice were divided into 4 equal groups:

(1) Graft group: transplantation of ovarian tissues with $10 \mu \mathrm{L}$ of Matrigel; (2) Graft + MSC group: each ovarian fragment was transplanted with $5 \times 10^{5}$ MSCs packaged in $10 \mu \mathrm{L}$ of Matrigel; (3) Graft + MSC + Control antibody $(\mathrm{Ab})$ group: each ovarian fragment was transplanted with $5 \times 10^{5}$ MSCs with $200 \mu \mathrm{g} / \mathrm{mL}$ of a control isotype IgG antibody (Thermo Fisher Scientific, Carlsbad, CA) packaged in $10 \mu \mathrm{L}$ of Matrigel; and (4) Graft + MSC + ANG $\mathrm{Ab}$ group: each ovarian fragment was transplanted with $5 \times 10^{5}$ MSCs with $200 \mu \mathrm{g} / \mathrm{mL}$ of the ANG monoclonal antibody 26-2 F (Millipore, Germany) packaged in $10 \mu \mathrm{L}$ of Matrigel.

The detailed transplantation procedure has been described previously [13]. Briefly, before transplantation, the SCID mice were anesthetized via intraperitoneal 
injection of 2,2,2-tribromoethyl alcohol (Sigma, St. Louis, MO) and tertamyl alcohol (Sigma, St. Louis, MO). Two skin incisions were made in the lower third of the abdominal wall on both sides after sterilizing the area. The ovarian tissues were randomly chosen and xenografted into the subcutaneous interspace, where the cortical side adhered to the skin and the medullar side adhered to the fascia. Two pieces of ovarian tissue were then placed in each mouse. The skin incisions were subsequently closed with absorbable 5/0 Prolene (Ethicon, Somerville, NJ). All procedures were performed under aseptic conditions. The animals were euthanized via cervical dislocation after 7 days (3 mice in each group). Ovarian cortical tissues were retrieved and fixed in $4 \%$ formaldehyde for histological and immunohistochemical examination.

\section{Human angiogenesis array}

A human antibody-based array kit was purchased from Raybiotech (Raybiotech Inc, Norcross GA); in the kit, glass slides were printed as sub-arrays consisting of 42 antigen-specific antibodies against angiogenesis-related factors. The antibody array was used according to the manufacturer's instructions. The results were normalized to internal positive controls for comparison. Two replicates per antibody were spotted, and the average of the median signal intensity from each spot (minus local background subtraction) was used for the calculation. The arrays were visualized using ImageQuant LAS4000 software (GE Healthcare) and analyzed using Image (National Institutes of Health).

\section{Assessment of ovarian histology}

Ovarian histology was evaluated based on hematoxylin and eosin (HE) staining. The detailed procedures have been described previously [13]. After fixation, xenografted ovarian tissues were processed for routine paraffin embedding, and 5 - $\mu \mathrm{m}$-thick serial sections were prepared. One from every five serial sections was used for HE staining, and follicles were counted in five randomly selected fields at $\times 400$ magnification. To avoid counting follicles more than once, only follicles with a visible nucleus were counted. Follicle stages were classified as previously described [30]. The number of primordial follicles in each group was expressed as the sum of the follicles in 25 different sections.

\section{Immunohistochemistry and Immunofluorescence study}

Immunohistochemistry was performed using the $A B C$ Staining System (Zhongshan Golden Bridge Biotechnology, Inc., Beijing, China). The detailed procedures have been described previously [31]. For CD-31 staining, human fetal villus tissues (10-week-old) were used as a positive control. For AC-3 staining, thawed human ovaries (female, age: 21 ) were used as a positive control. The primary antibody was omitted in the negative control. The follicles with positive staining (brown staining in the cytoplasm/nucleus) and the total number of follicles were counted in five random fields $(\times 400)$ of each section in 25 different sections in each sample. To evaluate vascular density, CD31-positive vessels were counted in five random fields ( $\times 400$ magnification) of each section and in 5 different sections from each sample. Immunofluorescence staining of CD31 and Ki67 were performed as described previously [32].

\section{Statistical analysis}

All experiments were repeated three times unless specified otherwise. Analyses were performed using SPSS 13.0 software (SPSS, Inc., Chicago, IL, USA). For the microarray, qPCR and ELISA data, statistical significance was determined with a two-tailed Student's $t$ test. The vascular density, follicle count and AC-3 percentage data were analyzed via one-way or two-way analysis of variance (ANOVA), followed by pairwise comparisons using the LSD method. All $P$ values were two-sided. A $P$ value $<0.05$ was considered statistically significant.

\section{Results}

ANG levels are significantly increased upon co-transplantation of MSCs with ovarian tissues

The characterization of MSCs was shown in Additional file 2: Figure S2. The isolated MSCs were negative for CD34, CD45, and CD19 and positive for CD44, CD90, and CD105. IHC study showed scattered positive expression of CD90 and CD105 in the ovarian tissue cotransplanted with MSCs (Additional file 3: Figure S3). To investigate whether certain secreted factors were specifically increased in the co-transplanted ovarian tissues and MSCs, we analyzed 42 angiogenesis-related proteins via protein microarray analysis (Fig. 1). The results were compared with the Graft group (xenografted ovarian tissues only). In total, the expression levels of 37 proteins were higher in the Graft + MSC group, whereas the expression levels of 5 proteins were higher in the Graft group. Twelve proteins were at least two-fold more abundant in co-transplanted ovarian tissue and MSCs than in solely ovarian grafts (Fig. 1b). The remaining proteins exhibited non-significant changes. By contrast, no proteins were at least twice as abundant in solely ovarian grafts as in co-transplanted ovarian tissue and MSCs. We were intrigued that ANG exhibited a marked increase in expression (up to 4.49-fold) in cotransplanted ovarian tissue and MSCs, particularly as it has been reported that ANG plays an essential role in endothelial cell proliferation and angiogenesis [33]. Although the expression of angiopoietin-2 was even higher (by up to 5.76-fold), angiopoietin-2 has been 
A

\begin{tabular}{|c|c|c|c|c|c|c|c|}
\hline POS-1 & POS-2 & POS-3 & NEG & NEG & Angiogenin & EGF & ENA-78 \\
\hline POS-1 & POS-2 & POS-3 & NEG & NEG & Angiogenin & EGF & ENA-78 \\
\hline GRO & IFN-gamma & IGF-1 & IL-6 & IL-8 & LEPTIN & MCP-1 & Endostatin \\
\hline GRO & IFN-gamma & IGF-I & IL-6 & IL-8 & LEPTIN & MCP-1 & Endostatin \\
\hline PIGF & RANTES & TGF-beta1 & TIMP-1 & TIMP-2 & Thrombopoietin & VEGF & IL-4 \\
\hline PIGF & RANTES & TGF-beta1 & TIMP-1 & TIMP-2 & Thrombopoietin & VEGF & IL-4 \\
\hline NEG & NEG & NEG & NEG & NEG & NEG & NEG & TNF-alpha \\
\hline NEG & NEG & NEG & NEG & NEG & NEG & NEG & TNF-alpha \\
\hline POS-1 & POS-2 & POS-3 & NEG & NEG & Angiopoietin-1 & Angiopoietin-2 & Angiostatin \\
\hline POS-1 & POS-2 & POS-3 & NEG & NEG & Angiopoietin-1 & Angiopoietin-2 & Angiostatin \\
\hline G-CSF & GM-CSF & I-309 & IL-10 & IL-1alpha & IL-1beta & IL-2 & PDGF-BB \\
\hline G-CSF & GM-CSF & I-309 & IL-10 & IL-1alpha & IL-1beta & IL-2 & PDGF-BB \\
\hline I-TAC & MCP-3 & MCP-4 & MMP-1 & MMP-9 & PECAM-1 & Tie-2 & VEGF-D \\
\hline I-TAC & MCP-3 & MCP-4 & MMP-1 & MMP-9 & PECAM-1 & Tie-2 & VEGF-D \\
\hline u PAR & VEGF R2 & VEGF R3 & NEG & NEG & NEG & NEG & NEG \\
\hline u PAR & VEGF R2 & VEGF R3 & NEG & NEG & NEG & NEG & NEG \\
\hline
\end{tabular}

B

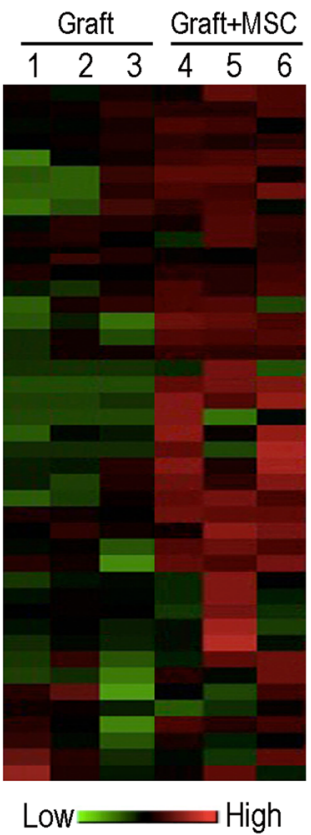

Abundant in Graft+MSC group

\begin{tabular}{ccc}
\hline Protein & Increased fold & P-value \\
Angiopoietin-2 & 5.76 & $<0.01$ \\
Angiogenin & 4.49 & 0.04 \\
Endostatin & 4.28 & $<0.01$ \\
IL-1beta & 4.2 & 0.08 \\
PECAM-1 & 3.85 & 0.09 \\
MMP-9 & 3.52 & 0.18 \\
u PAR & 3.47 & 0.12 \\
TIMP-2 & 3.22 & 0.01 \\
Thrombopoietin & 3.11 & 0.02 \\
Angiostatin & 3.07 & 0.04 \\
MCP-3 & 3.07 & 0.26 \\
MCP-4 & 2.33 & 0.35
\end{tabular}

Abundant in Graft group

\begin{tabular}{ccc}
\hline Protein & Increased fold & P-value \\
MMP-1 & 1.54 & 0.40 \\
IFN-gamma & 1.52 & 0.49 \\
TGF-beta1 & 1.19 & 0.50 \\
GRO & 1.14 & 0.76 \\
IL-6 & 1.12 & 0.38
\end{tabular}

Fig 1 Angiogenin level is significantly increased in co-transplanted of ovarian tissues and MSCs. a List of antibodies against angiogenesis related cytokine factors by RayBiotech human antibody array; b Comparison of the panel of protein expression profiles in ovarian graft and MSCs cOtransplantation (Graft + MSC group) versus Graft group. (Left) Heat maps were developed with the hierarchical clustering algorithm between the two groups. For each protein, signals from all conditions were averaged to generate the baseline. Signals above baseline are red; signals below baseline are green. The heat map key shows log2-fold changes from baseline. (Right) Lists of differential expressed protein that was abundant in Graft + MSC group or in MSC group. MSC, mesenchymal stem cells. POS, positive control. NEG, negative control

associated with endothelial cell death and vascular regression [34]. Therefore, we focused on ANG in our subsequent experiments.

\section{Blockade of ANG suppresses MSC-stimulated ovarian angiogenesis}

Because ANG was robustly increased in MSCs cotransplanted with ovarian grafts, we speculated that ANG might play a key role in angiogenesis and follicle survival in MSCs co-transplanted with ovarian grafts. To test our hypothesis, we specifically inhibited ANG function in MSCs using either shANG or an anti-ANG neutralizing antibody. The results of RT-PCR indicated that shANG significantly suppressed the mRNA expression of ANG, with a $72.4 \%$ knockdown efficiency $(P<0.01$, Additional file 4: Figure S4A). In addition, secreted ANG was measured in the cell culture media using a commercial ELISA. As expected, a significantly lower secreted ANG level was observed in the shANG-transfected MSCs than in the shCTRL-transfected MSCs $(P<0.01$, Additional file 4: Figure S4B).

Ovarian angiogenesis in each group in the shRNA and antibody blockade experiments was assessed via CD31 staining and microvascular density counts (Fig. 2a, c). Similar to our previous study, by day 7, cotransplantation of MSCs with the graft (Graft + MSC group) had significantly promoted the density of CD31positive microvessels compared with the densities in the 

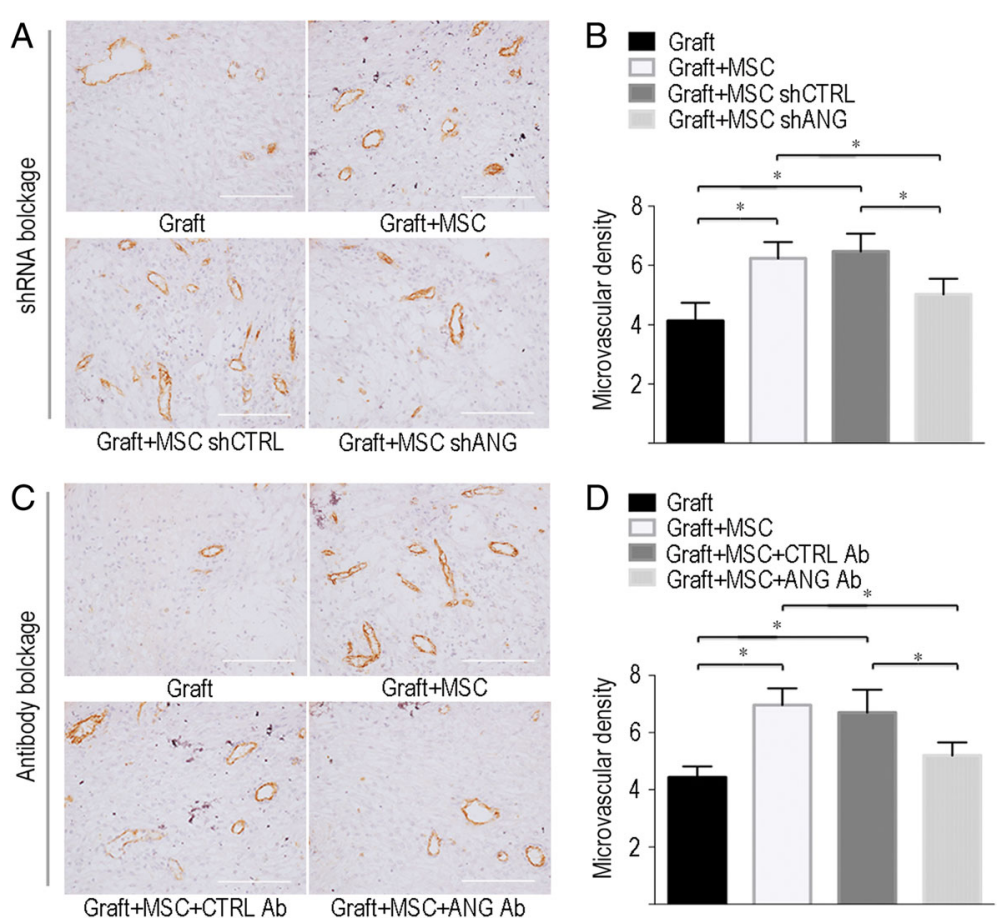

Fig 2 Angiogenin-blockade suppressed MSCs-stimulated ovarian angiogenesis. a Representative figures showing microvascular density of ovarian tissues in shRNA blockage analysis, as determined by CD31 immunoactivity, at post-transplantation day-7 in each group. b Quantitative analysis of microvascular densities between different groups in shRNA blockage analysis, $\mathbf{c}$ Representative figures showing microvascular density of ovarian tissues in antibody blockage experiment, as determined by CD31 immunoactivity, at post-transplantation day-7 in each groups. $\mathbf{d}$ Quantitative analysis of microvascular density between different groups in antibody blockage experiment. Data shown are means \pm SEM of triplicates in a representative experiment. MSC, mesenchymal stem cells; ANG, angiogenin; CTRL, control; Ab, antibody; shANG, ANG specific short hairpin RNA; shCTRL, control short hairpin RNA, ${ }^{*} P<0.05$. Scale bar $=100 \mu \mathrm{m}$

Graft group (Additional file 5: Figure S5) in both the shRNA (6.23 \pm 0.55 vs. $4.13 \pm 0.61$, respectively, $P<0.05$, Fig. 2b) and ANG antibody blockade experiments (6.97 \pm 0.59 vs. $4.43 \pm 0.38$, respectively, $P<0.05$, Fig. 2 d). In the shRNA blockade experiment, the microvascular density was significantly decreased in the Graft + MSC shANG group $(5.03 \pm 0.51)$ compared with those of the Graft + MSC (6.23 $\pm 0.55, P<0.05$, Fig. 2b) and Graft + MSC shCTRL groups $(6.47 \pm 0.60, P<0.05$, Fig. $2 b)$. This finding showed that ovarian angiogenesis induced by MSCs was significantly suppressed by shANG, most likely due to the suppression of ANG secretion. Although shCTRL did not suppress MSC-induced angiogenesis, the microvascular density in the Graft + MSC shCTRL groups $(6.47 \pm 0.60)$ was not significantly different from that in the Graft + MSC groups $(6.23 \pm 0.55, P=0.63$, Fig. $2 b)$. In the antibody blockade experiment, we found that the microvascular density was significantly decreased in the Graft + MSC + ANG Ab group $(5.2 \pm 0.46)$ compared with those in the Graft + MSC group $(6.97 \pm 0.59, P<0.05$, Fig. 2d) and the Graft + MSC + CTRL Ab group (6.70 \pm $0.80, P<0.05$, Fig. $2 \mathrm{~d}$ ), which showed that the ANG Ab sufficiently blocked MSC-induced angiogenesis, probably via the neutralization of ANG secretion. By contrast, the CTRL IgG Ab had no influence on the effect of MSCs on ovarian angiogenesis, as the microvascular density in the Graft + MSC + CTRL Ab group (6.70 \pm 0.80$)$ was not significantly different from that in the Graft + MSC group $(6.97 \pm 0.59, P=0.45$, Fig. $2 d)$.

\section{Blockade of ANG neutralizes MSC-stimulated follicular survival}

Before transplantation, the average number of primodial follicles in ovarian tissue is $63.17 \pm 9.52$. Next we examined the number of primordial follicles in xenografted ovarian tissues after ANG blockade in MSCs using either shRNA or a neutralizing antibody. Representative HEstained follicles from each group are shown in Fig. 3 and Additional file 6: Figure S6. Consistent with our previous study, the co-transplantation of MSCs with ovarian tissues (Graft + MSC group) significantly increased the follicle count compared with that of the Graft group in both the shRNA $(36.27 \pm 2.85$ vs. $27.13 \pm 2.32$, respectively, $P<0.05$, Fig. $3 \mathrm{~b})$ and antibody blockade experiments ( $34.57 \pm 1.60$ vs. $26.73 \pm 2.07$, respectively, $P<0.05$, Fig. $3 \mathrm{~d}$ ). In the shRNA experiment, the numbers of primordial follicles in the Graft + MSC shANG 

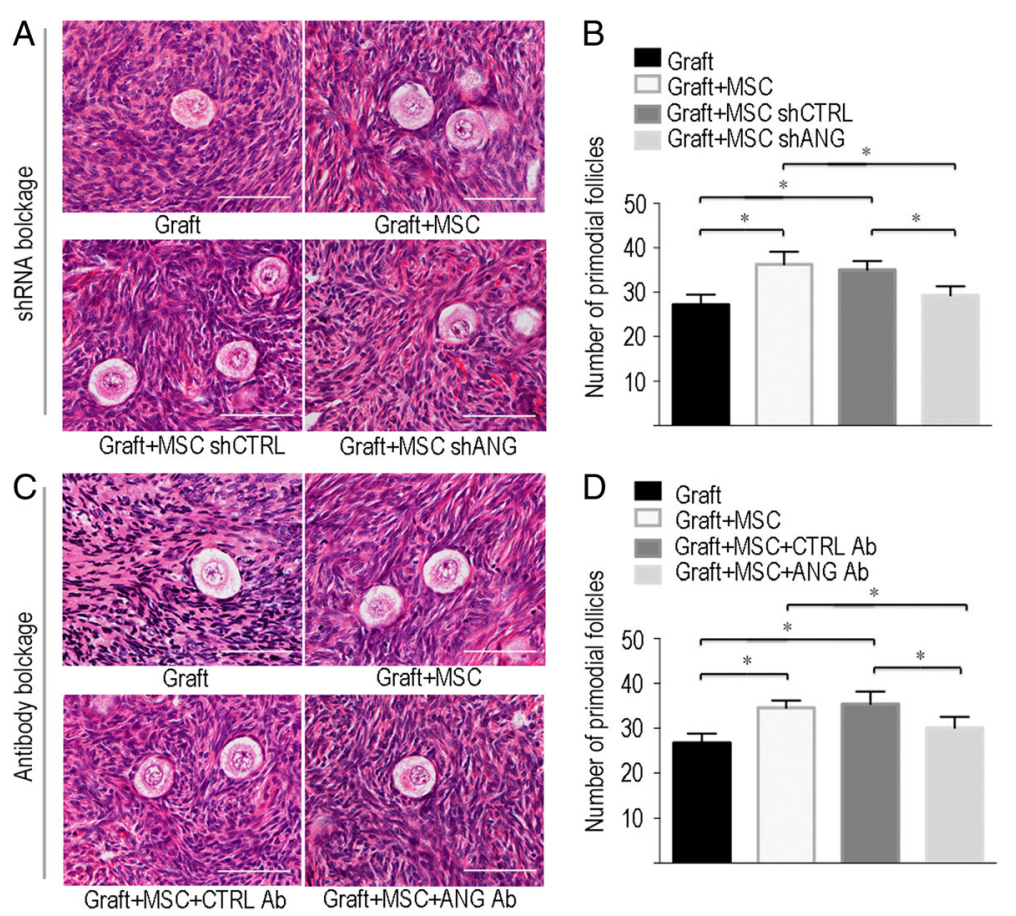

Fig 3 Blockade of angiogenin neutralized MSC-stimulated follicular survival after co-transplantation of MSCs. a Representative images of HE-stained section showing number of primodial follicles in ovarian tissue at post-transplant day-7 of each group in the shANG blockage experiment. b Quantitative analysis of follicular numbers in different groups in shANG blockage experiment. $\mathbf{c}$ Representative images of HE-stained section showing number of primodial follicles in ovarian tissue at post-transplant day-7 of each group in antibody blockage experiment. $\mathbf{d}$ Quantitative analysis of primodial follicular numbers in different groups in antibody blockage experiment. Data shown are means \pm SEM of triplicates in a representative experiment. MSC, mesenchymal stem cells; ANG, angiogenin; CTRL, control; Ab, antibody; shANG, ANG specific short hairpin RNA; shCTRL, control short hairpin $\mathrm{RNA}^{*} P<0.05$. Scale bar $=50 \mu \mathrm{m}$

group $(29.27 \pm 2.05)$ were significantly lower than those in the Graft $+\operatorname{MSC}(36.27 \pm 2.85, P<0.05$, Fig. 3b) and the Graft + MSC shCTRL groups $(35.03 \pm 1.99, \quad P<0.05$, Fig. 3b). This result indicates that the suppression of ANG secretion by shANG significantly neutralized the ability of MSCs to enhance follicular survival. However, shCTRL did not attenuate the effect of MSCs on the enhancement of follicular survival, as the numbers of primordial follicles in the Graft + MSC + CTRL Ab group (35.03 \pm 1.99$)$ was not significantly different from that in the Graft + MSC group ( $36.27 \pm 2.85$, Fig. $3 \mathrm{~b})$. In the antibody blockade experiment, the number of primordial follicles in the Graft + MSC + ANG Ab group (30.10 \pm 2.43$)$ was significantly lower than those in the Graft + MSC (34.57 $\pm 1.60, P<0.05$, Fig. $3 \mathrm{~d})$ and Graft + MSC + CTRL Ab groups $(35.40 \pm 2.78, P<0.05$, Fig. $3 \mathrm{~d})$, indicating that the suppression of ANG secretion by the ANG antibody significantly neutralized the ability of MSCs to enhance follicular survival. However, the CTRL IgG $\mathrm{Ab}$ did not have a neutralizing effect, as the number of primordial follicles in the Graft + MSC + CTRL Ab groups $(35.40 \pm 2.78)$ was not significantly different from that in the Graft + MSC group $(34.57 \pm 1.60, P=$ 0.66, Fig. 3d).

\section{Blockade of ANG increases follicular apoptosis after the co-transplantation of MSCs}

Active caspase 3 (AC-3) was used as a marker of apoptosis in IHC experiments, and representative images of AC-3 expression in each group are presented in Fig. 4a and c. Similar to our previous study, MSC cotransplantation significantly reduced follicular apoptosis in the Graft + MSC group compared with that in the Graft group in both the shRNA $(12.3 \pm 2.5 \%$ vs. $18.7 \pm$ $3.3 \%$, respectively, $P<0.05$. Fig. $4 \mathrm{~b})$ and antibody blockade experiments $(9.7 \pm 3.4 \%$ vs. $20.0 \pm 3.1 \%$, respectively, $P<0.05$. Fig. 4d). We hypothesized that ANG blockade may increase follicular apoptosis with co-transplantation of MSCs. Indeed, in the shRNA blockade experiment, shANG significantly increased follicular apoptosis, as a significantly elevated number of AC-3-positive primordial follicles was observed in the Graft + MSC shANG group $(17.2 \pm 2.4 \%)$ in comparison to the number in the Graft + MSC $(12.3 \pm 2.5 \%, P<0.05$. Fig $4 \mathrm{~b})$ and the Graft + shCTRL MSC groups $(11.2 \pm 2.0 \%, P<0.05$. Fig $4 \mathrm{~b})$. It is not surprising that shCTRL did not affect follicular apoptosis in xenografted ovarian tissues after the cotransplantation of MSCs, as the follicular apoptosis rate in the Graft + MSC shCTRL group (11.2 $\pm 2.0 \%)$ was not 

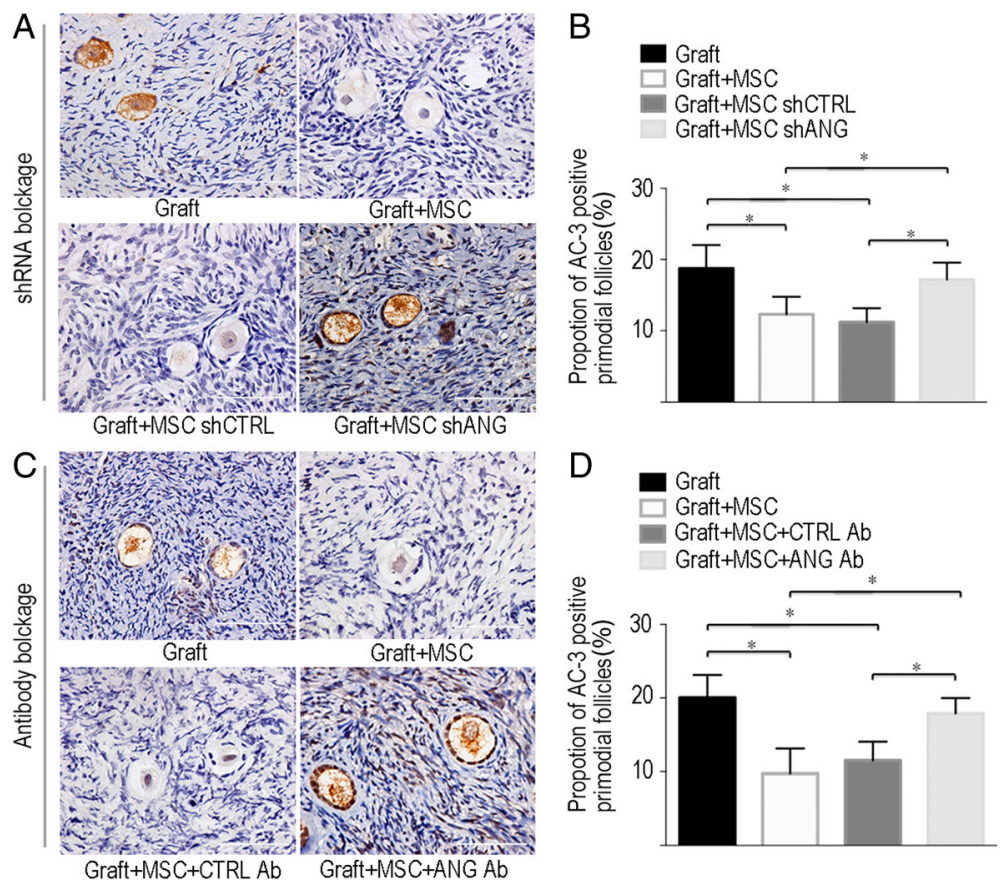

Fig 4 Blockade of angiogenin increased primordial follicular apoptosis after co-transplantation of MSC. a Representative images showing primordial follicular apoptosis as determined by AC3 staining of ovarian tissues at post-transplantation day-7 in different groups in shANG blockage experiment. b Quantitative analysis of primordial follicular apoptosis between different groups in shANG blockage experiment. c Representative images showing primordial follicular apoptosis as determined by AC3 staining of ovarian tissues at post-transplantation day-7 in different groups in antibody blockage experiment. d Quantitative analysis of primordial follicular apoptosis between different groups in antibody blockage experiment. Data shown are means \pm SEM of triplicates in a representative experiment. MSC, mesenchymal stem cells; ANG, angiogenin; Ab, antibody, shANG, ANG specific short hairpin RNA, shCTRL, control short hairpin RNA, ${ }^{*} P<0.05$. Scale bar $=50 \mu \mathrm{m}$

significantly different from that of the Graft + MSC group (12.3 $\pm 2.5 \%, P=0.61$, Fig. $4 \mathrm{~b})$.

In the antibody blockade experiment, the ANG antibody significantly increased follicular apoptosis, and significantly elevated numbers of AC-3-positive follicles were observed in the Graft + MSC + ANG Ab group $(17.9 \pm 2.1 \%)$ compared with the numbers in the Graft + MSC group $(9.7 \pm 3.4 \%, P<0.05$. Fig $4 \mathrm{~d})$ and the Graft + MSC + CTRL Ab group ( $11.5 \pm 2.6 \%, P<0.05$. Fig $4 d)$. It is not surprising that the CTRL IgG Ab did not affect follicular apoptosis in xenografted ovarian tissues after the co-transplantation of MSCs; the follicular apoptosis rate in the Graft + MSC + CTRL Ab group (11.5 $\pm 2.6 \%)$ was not significantly different from that of the Graft + MSC group (9.7 $\pm 3.4 \%, P=0.47$, Fig. 4 d).

\section{Discussion}

Our study highlights several important findings. The first is that the expression levels of a panel of proteins, including ANG, were significantly increased upon the co-transplantation of MSCs with ovarian tissues. The second is that the blockade of ANG using either the specific shANG or an ANG neutralizing antibody could suppress MSC-induced ovarian angiogenesis and follicle survival. These results indicated that MSC-derived ANG induced angiogenesis and follicle survival in xenografted human ovarian tissues. Our study is the first to demonstrate the crucial role of ANG in mediating the proangiogenic effect of MSCs co-transplanted with ovarian tissue, and these results provide a theoretical basis for further research on and the application of MSCs in ovarian tissue transplantation.

The ability of MSCs to release paracrine factors with pro-angiogenic functions has been observed in many studies. Umbilical cord MSC-derived microvesicles containing several angiogenic factors, including EGF and VEGF, contribute to the pro-angiogenic effect of MSCs [35]. Placental chorionic villus MSCs showing a high capacity to release angiogenic factors, including VEGF and hepatocyte growth factor, have been found to contribute to the functional improvement of ischemic hind limbs of nude mice after transplantation [36]. Preclinical and clinical studies have also shown that MSCs can augment cardiac function upon implantation into the ischemic/infarcted myocardium [37, 38]. These studies have suggested that pro-angiogenic factors released by MSCs could stimulate angiogenesis and increase regional perfusion. In our model of MSC and ovarian tissue co-transplantation, MSCs were packaged in Matrigel surrounding the ovarian graft, providing a suitable 
microenvironment for the release of pro-angiogenic proteins. Antibody-based cytokine array technology offers a novel approach for gaining insight into changes in protein expression profiles. Our array results reflected the secretion profiles of MSCs co-transplanted with ovarian tissues. Further analyses showed that despite the variety of angiogenic factors in the paracrine profiles of MSCs, the targeted inhibition of ANG had a substantial effect on the angiogenic ability of MSCs in ovarian transplantation. One possible explanation for this finding is that, as previously reported, the presence of ANG could be essential for other angiogenic factors, including VEGF and bFGF, to functionally induce angiogenesis [33].

ANG, also known as ribonuclease 5 , is a secreted protein. In the past several years, studies have shown that ANG has angiogenic [39], neurogenic [40], and immuneregulatory [41] functions. Several pro-angiogenic factors, including ANG, have been isolated from the follicular fluid, and a positive association with follicular growth has also been suggested [42-45]. The present study suggests a critical role for ANG in establishing vascularization and promoting follicle survival in ovarian grafts cotransplanted with MSCs. It should be acknowledged that angiogenesis is a complicated process and that the proangiogenic effects of MSCs involve a variety of secreted factors. In our study, after the blockade of ANG, the proangiogenic effect of MSCs was attenuated. This inhibition was not complete, as the microvascular density after either shRNA or antibody blockade was still higher than in the Graft group; however, this difference did not reach statistical significance. This phenomenon might be explained by the fact that other factors independent of ANG may also be involved in the pro-angiogenic effect of MSCs. Interestingly, the array result suggests induced expression of both MMPs and TIMP-2 in MSCs transplanted ovary. MMPs were speculated to be induced by the hypoxic condition of ovarian grafts. Since TIMP-2 is an known antagonist of MMPs and endogenous inhibitor of angiogenesis, the induction of TIMP-2 may suppress excess formation of microvessels and non-functional microvessels. Additionally, it is worth noting that other secreted factors that have been reported to exhibit an anti-angiogenic capacity, such as angiopoietin-2, were significantly increased, as well, according to our microarray results. This result might be attributed to the equilibrium between angiogenic stimulators and inhibitors in the angiogenically balanced microenvironment established by the MSCs, which precisely regulates the rate of blood vessel formation.

Ischemia-reperfusion injury after ovarian tissue transplantation has been shown to lead to considerable follicular loss and to shorten the duration of ovarian function [46]. Graft angiogenesis following ovarian tissue transplantation is critical for graft viability. The reestablishment of vascularization in xenografted ovarian tissues is observed within $48 \mathrm{~h}$ of grafting, and this process lasts for approximately 1 week [47]. In an ovarian autograft study using mice, blood perfusion could be observed on day 3 after transplantation, and functional vessels could be detected on day 7 post-transplantation [48, 49]. Similarly, in a human study, microvessels started to form $48 \mathrm{~h}$ post-transplantation, whereas functional vessels required approximately 7 days to generate; thus, 7 days was proposed as an important landmark after transplantation $[31,50]$. Therefore, in the present study, xenografted ovarian tissues were retrieved on day 7 post-transplantation. We selected the subcutaneous route for transplantation in this study since the subcutaneous areas of the abdominal wall have been reported to be one of the most preferable options for follicular development in ovarian transplantation [51, 52].

In recent years, numerous studies have focused on interventions to facilitate angiogenesis in transplanted ovarian tissue. Labied et al. [53] suggested that VEGF111 could stimulate vascular endothelial cell proliferation and functional angiogenesis, thereby increasing the viability of the ovarian cortex. Experimental data have also shown that basic fibroblast growth factor and fibrin hydrogel can improve angiogenesis and promote follicle development in mice [31]. However, none of these factors have been successfully utilized in a clinical setting thus far. Further investigation of methods for improving angiogenesis in ovarian tissue transplantation is required. Our previous study showed that MSCs show promise for the improvement of ovarian tissue transplantation and follicle growth $[13,26]$. Although MSCs can be obtained from bone marrow, readily cultured and preserved for future use, further research is needed to develop methods for their use. Our findings suggest mechanistic explanations for the angiogenic effect of MSCs in ovarian transplantation, providing important information for further applications.

Previous studies have indicated that the therapeutic application of secreted molecules as a possible replacement for stem cells might lead to the development of safe and effective therapeutic strategies with predictable outcomes [22]. Therefore, practical interventions to facilitate angiogenesis, including treatment with exogenous angiogenic factors, might be promising in clinical settings, as this approach will circumvent the ethical and safety constraints associated with the direct use of human MSCs. In our preliminary study, the treatment of xenografted ovarian tissues with exogenous recombinant ANG enhanced angiogenesis, even without MSCs (data not shown). However, due to the scarcity of human ovarian tissue samples available for research, we were unable to comprehensively investigate the outcomes of ovarian transplantation through treatment with exogenous recombinant ANG protein. Additional studies of the 
use of human ovarian cortical grafts with long-term ANG treatment are necessary to elucidate the effects and functional mechanism of ANG in mediating the angiogenesis of xenografted ovarian tissue. Continuing research on this topic will help elucidate the efficacy and safety of exogenous ANG intervention in ovarian tissue transplantation in clinical settings.

\section{Conclusions}

In conclusion, our findings are the first to suggest that MSCs mediate angiogenesis and follicle survival in xenografted human ovarian tissue through ANG. Our results provide a theoretical basis and important information for the further application of MSCs in ovarian tissue transplantation and could eventually aid patients in preserving fertility.

\section{Additional files}

Additional file 1: Figure S1. Isolation of MSCs from human bone

Additional file 2: Figure S2. Identification of MSCs by Flow Cytometry. (A) Representative histogram of FACS results showing the MSCs surface marker profile. The blue peak indicated the specific antibodies: CD34, CD45, CD19, negative cocktail (including CD44, CD90 and CD105). The red peak represented the isotope antibodies. (B) Positive expression of CD34, CD45, CD19 and negative cocktail (including CD44, CD90 and CD105) in five individuals as examined by flow cytometry was expressed as mean \pm SD. The proportion of cells expressing CD44, CD90, CD105 and negative cocktail, which were analyzed from 5 independent samples, were $96.6 \% \pm 2.1 \%, 96.4 \% \pm 2.2 \%, 97.2 \% \pm 2.0 \%$ and $2.9 \% \pm 0.6 \%$. (JPG $418 \mathrm{~kb}$ )

Additional file 3: Figure S3. Representative images showing expression of CD90 and CD105 in ovarian sections after co-transplantation of MSCs in both high and low magnification. MSC, mesenchymal stem cells. Scale bar $=200 \mu \mathrm{m}$ in A and C, Scale bar $=50 \mu \mathrm{m}$ in B and D. (JPG $453 \mathrm{~kb}$ )

Additional file 4: Figure S4. The MSC clones stably knocking down ANG were identified and verified on qPCR and ELISA analysis. A) Results of quantitative PCR showed a significant knock-down of ANG mRNA expression in the shANG transfected MSCs $(n=3)$. B) Secreted ANG protein level in the shANG and shCTRL transfected MSCs groups were determined by ELISA $(n=3)$. Data are shown as means \pm SEM of triplicates in a representative experiment. MSC, mesenchymal stem cells; ANG, angiogenin; shANG, ANG specific short hairpin RNA, shCTRL: control short hairpin RNA, * $P<0.01$. (TIF $7881 \mathrm{~kb}$ )

Additional file 5: Figure S5. Representative images showing triple staining of Ki67, DAPI and CD31 in ovarian graft with or without co-transplantation of MSCs. Vasculature is shown in red, cell nuclei are shown in blue and Ki67 positive nuclei are shown in green. Scale bar $=50 \mu \mathrm{m}$. (JPG $299 \mathrm{~kb}$ )

Additional file 6: Figure S6. Representative images showing HE staining of ovarian sections in ovarian graft with or without co-transplantation of MSCs in both high and low magnification. MSC, mesenchymal stem cells. Scale bar $=200 \mu \mathrm{m}$ in A and C, Scale bar $=50 \mu \mathrm{m}$ in B and D. (JPG $507 \mathrm{~kb}$ )

\section{Abbreviations}

Ab: Antibody; ANG: Angiogenin; CTRL: control; MSC: Mesenchymal stem cells; shANG: ANG specific short hairpin RNA; shCTRL: Control short hairpin RNA; shRNA: Short hairpin RNA

\section{Acknowledgements}

The authors thank Dr. Jijun Wang and Lei Tian for their support in collecting bone marrow tissue. The authors are grateful to Dr. Liyuan Tao for technical support in statistical analyses.

\section{Funding}

This work was supported by the National Science Foundation of China (No. 31230047; No. 81471508; No. 81571386;No. 81471427;No.31429004; No. 81300456), National Key Technology R\&D Program of China (No. 2015BAI13B06, 2014BAI05B04), Guangdong Natural Science Foundation (2014A03031379),Guangdong Medical Scientific Foundation (A2014665) and Research Fund for Distinguished Experts, Guangxi, China.

\section{Availability of data and materials}

The datasets used and/or analysed during the current study available from the corresponding author on reasonable request.

\section{Authors' contributions}

$Y Z$ and $X X$ wrote the manuscript. $X X, J Y$ and JQ designed the experiments; $Y Z, J Y, T W, T Y$ and $C L$ performed the experiments; $X Z 、 R L$ and HMC participated in the analysis and discussion of the results. All authors read and approved the final version of the manuscript.

\section{Competing interests}

The authors declare that they have no competing interests.

\section{Consent for publication}

Not applicable.

\section{Ethics approval}

All experiments were performed in strict accordance with the Ethics Committee at the Peking University Third Hospital. Informed consent was obtained from all subjects. The Institutional Committee of the Peking University approved the experimental protocols (registration number: 2009005). All efforts were made to minimize the number of animals used and their suffering.

\section{Author details}

${ }^{1}$ Department of Obstetrics and Gynecology, Center for Reproductive Medicine, Peking University Third Hospital, No.49 North HuaYuan Road, HaiDian District, Beijing 100191, China. ${ }^{2}$ Beijing Key Laboratory of Reproductive Endocrinology and Assisted Reproduction, Beijing 100191, China. ${ }^{3}$ Key Laboratory of Assisted Reproduction, Ministry of Education, Beijing 100191, China. ${ }^{4}$ Department of Obstetrics and Gynecology, Center for Reproductive Medicine, Peking University Shenzhen Hospital, No.1120 Lotus Road, FuTian District, Shenzhen, Guangdong 518000, China. ${ }^{5}$ Department of Obstetrics and Gynecology, Center for Reproductive Medicine, Shengjing Hospital of China Medical University, Shenyang 100004, China. ${ }^{6}$ Department of Obstetrics and Gynaecology, Child and Family Research Institute, University of British Columbia, Vancouver V5Z4H4, Canada.

Received: 8 November 2016 Accepted: 23 February 2017 Published online: 09 March 2017

\section{References}

1. Qiao J, Li R. Fertility preservation: challenges and opportunities. Lancet. 2014;384:1246-7.

2. Bastings L, Beerendonk CC, Westphal JR, Massuger LF, Kaal SE, van Leeuwen FE, Braat DD, Peek R. Autotransplantation of cryopreserved ovarian tissue in cancer survivors and the risk of reintroducing malignancy: a systematic review. Hum Reprod Update. 2013;19:483-506.

3. Grynberg M, Poulain M, Sebag-Peyrelevade S, le Parco S, Fanchin R, Frydman N. Ovarian tissue and follicle transplantation as an option for fertility preservation. Fertil Steril. 2012;97:1260-8.

4. Gracia CR, Chang J, Kondapalli L, Prewitt M, Carlson CA, Mattei P, Jeffers S, Ginsberg JP. Ovarian tissue cryopreservation for fertility preservation in cancer patients: successful establishment and feasibility of a multidisciplinary collaboration. J Assist Reprod Genet. 2012;29:495-502.

5. Fabbri R, Vicenti R, Magnani V, Pasquinelli G, Macciocca M, Parazza I, Paradisi $\mathrm{R}$, Battaglia C, Venturoli S. Cryopreservation of ovarian tissue in breast cancer patients: 10 years of experience. Future Oncol. 2012;8:1613-9. 
6. Lambertini M, Del Mastro L, Pescio MC, Andersen CY, Azim Jr HA, Peccatori FA, Costa M, Revelli A, Salvagno F, Gennari A, et al. Cancer and fertility preservation: international recommendations from an expert meeting. BMC Med. 2016;14:1

7. Yang H, Lee HH, Lee HC, Ko DS, Kim SS. Assessment of vascular endothelial growth factor expression and apoptosis in the ovarian graft: can exogenous gonadotropin promote angiogenesis after ovarian transplantation? Fertil Steril. 2008;90:1550-8.

8. Van Eyck AS, Bouzin C, Feron O, Romeu L, Van Langendonckt A, Donnez J, Dolmans MM. Both host and graft vessels contribute to revascularization of xenografted human ovarian tissue in a murine model. Fertil Steril. 2010;93: 1676-85.

9. Abtahi NS, Eimani H, Vosough A, Shahverdi A, Fathi R, Hayati N, Nasiri N. Effect of therapeutic ultrasound on folliculogenesis, angiogenesis and apoptosis after heterotopic mouse ovarian transplantation. Ultrasound Med Biol. 2014;40:1535-44.

10. Hu H, Zou C. Mesenchymal stem cells in renal ischemia-reperfusion injury: Biological and therapeutic perspectives. Curr Stem Cell Res Ther. 2016;24. [Epub ahead of print]

11. Spees $\lrcorner$, Lee RH, Gregory CA. Mechanisms of mesenchymal stem/stromal cell function. Stem Cell Res Ther. 2016;7:125

12. Sherman LS, Shaker M, Mariotti $V$, Rameshwar P. Mesenchymal stromal/stem cells in drug therapy: New perspective. Cytotherapy. 2017;19(1):19-27.

13. Xia X, Yin T, Yan J, Yan L, Jin C, Lu C, Wang T, Zhu X, Zhi X, Wang J, et al. Mesenchymal Stem Cells Enhance Angiogenesis and Follicle Survival in Human Cryopreserved Ovarian Cortex Transplantation. Cell Transplant. 2015; 24:1999-2010.

14. Foronjy RF, Majka SM. The potential for resident lung mesenchymal stem cells to promote functional tissue regeneration: understanding microenvironmental cues. Cells. 2012;1:874.

15. Au P, Tam J, Fukumura D, Jain RK. Bone marrow-derived mesenchymal stem cells facilitate engineering of long-lasting functional vasculature. Blood. 2008;111:4551-8.

16. Lee RH, Seo MJ, Reger RL, Spees JL, Pulin AA, Olson SD, Prockop DJ. Multipotent stromal cells from human marrow home to and promote repair of pancreatic islets and renal glomeruli in diabetic NOD/scid mice. Proc Natl Acad Sci U S A. 2006;103:17438-43.

17. Liu SH, Huang JP, Lee RK, Huang MC, Wu YH, Chen CY, Chen CP. Paracrine factors from human placental multipotent mesenchymal stromal cells protect endothelium from oxidative injury via STAT3 and manganese superoxide dismutase activation. Biol Reprod. 2010;82: 905-13.

18. Sorrell JM, Baber MA, Caplan Al. Influence of adult mesenchymal stem cells on in vitro vascular formation. Tissue Eng Part A. 2009;15:1751-61.

19. Duffy GP, Ahsan T, O'Brien T, Barry F, Nerem RM. Bone marrowderived mesenchymal stem cells promote angiogenic processes in a time- and dose-dependent manner in vitro. Tissue Eng Part A. 2009; 15:2459-70

20. Wu Y, Chen L, Scott PG, Tredget EE. Mesenchymal stem cells enhance wound healing through differentiation and angiogenesis. Stem Cells. 2007; 25:2648-59.

21. Caplan Al, Correa D. The MSC: an injury drugstore. Cell Stem Cell. 2011;9:11-5

22. Kupcova Skalnikova H. Proteomic techniques for characterisation of mesenchymal stem cell secretome. Biochimie. 2013;95:2196-211.

23. Bronckaers A, Hilkens P, Martens W, Gervois P, Ratajczak J, Struys T, Lambrichts I. Mesenchymal stem/stromal cells as a pharmacological and therapeutic approach to accelerate angiogenesis. Pharmacol Ther. 2014;143: 181-96.

24. Hsieh JY, Wang HW, Chang SJ, Liao KH, Lee IH, Lin WS, Wu CH, Lin WY, Cheng SM. Mesenchymal stem cells from human umbilical cord express preferentially secreted factors related to neuroprotection, neurogenesis, and angiogenesis. PLoS One. 2013;8:e72604

25. Aranha AM, Zhang Z, Neiva KG, Costa CA, Hebling J, Nor JE. Hypoxia enhances the angiogenic potential of human dental pulp cells. J Endod. 2010;36:1633-7

26. Xia $X$, Wang $T$, Yin T, Yan L, Yan J, Lu C, Zhao L, Li M, Zhang Y, Jin H, et al. Mesenchymal Stem Cells Facilitate In Vitro Development of Human Preantral Follicle. Reprod Sci. 2015;22:1367-76.

27. Ernst $E$, Bergholdt $S$, Jorgensen JS, Andersen CY. The first woman to give birth to two children following transplantation of frozen/thawed ovarian tissue. Hum Reprod. 2010;25:1280-1.
28. Yoshimatsu G, Sakata N, Tsuchiya H, Minowa T, Takemura T, Morita H, Hata T. Fukase M, Aoki T, Ishida M, et al. The co-transplantation of bone marrow derived mesenchymal stem cells reduced inflammation in intramuscular islet transplantation. PLoS One. 2015;10:e0117561.

29. Ahn SY, Chang YS, Sung DK, Sung SI, Yoo HS, Im GH, Choi SJ, Park WS. Optimal Route for Mesenchymal Stem Cells Transplantation after Severe Intraventricular Hemorrhage in Newborn Rats. PLoS One. 2015;10:e0132919.

30. Gougeon A, Testart J. Germinal vesicle breakdown in oocytes of human atretic follicles during the menstrual cycle. J Reprod Fertil. 1986;78:389-401.

31. Gao JM, Yan J, Li R, Li M, Yan LY, Wang TR, Zhao HC, Zhao Y, Yu Y, Qiao J. Improvement in the quality of heterotopic allotransplanted mouse ovarian tissues with basic fibroblast growth factor and fibrin hydrogel. Hum Reprod. 2013:28:2784-93.

32. Niu G, Sun X, Cao Q, Courter D, Koong A, Le QT, Gambhir SS, Chen X. Cetuximab-based immunotherapy and radioimmunotherapy of head and neck squamous cell carcinoma. Clin Cancer Res. 2010;16:2095-105.

33. Kishimoto K, Liu S, Tsuji T, Olson KA, Hu GF. Endogenous angiogenin in endothelial cells is a general requirement for cell proliferation and angiogenesis. Oncogene. 2005;24:445-56.

34. Fagiani E, Christofori G. Angiopoietins in angiogenesis. Cancer Lett. 2013; 328:18-26.

35. Chen J, Liu Z, Hong MM, Zhang H, Chen C, Xiao M, Wang J, Yao F, Ba M, Liu $J$, et al. Proangiogenic compositions of microvesicles derived from human umbilical cord mesenchymal stem cells. PLoS One. 2014;9:e115316.

36. Du W, Li X, Chi Y, Ma F, Li Z, Yang S, Song B, Cui J, Ma T, Li J, et al. VCAM-1 + placenta chorionic villi-derived mesenchymal stem cells display potent pro-angiogenic activity. Stem Cell Res Ther. 2016;7:49.

37. Liu XH, Bai CG, Xu ZY, Huang SD, Yuan Y, Gong DJ, Zhang JR. Therapeutic potential of angiogenin modified mesenchymal stem cells: angiogenin improves mesenchymal stem cells survival under hypoxia and enhances vasculogenesis in myocardial infarction. Microvasc Res. 2008;76:23-30.

38. Huang SD, Lu FL, XU XY, Liu XH, Zhao XX, Zhao BZ, Wang L, Gong DJ, Yuan $Y, X u Z Y$. Transplantation of angiogenin-overexpressing mesenchymal stem cells synergistically augments cardiac function in a porcine model of chronic ischemia. J Thorac Cardiovasc Surg. 2006;132:1329-38.

39. Fett JW, Strydom DJ, Lobb RR, Alderman EM, Bethune JL, Riordan JF, Vallee BL. Isolation and characterization of angiogenin, an angiogenic protein from human carcinoma cells. Biochemistry. 1985:24:5480-6.

40. Subramanian $V$, Feng $Y$. A new role for angiogenin in neurite growth and pathfinding: implications for amyotrophic lateral sclerosis. Hum Mol Genet. 2007;16:1445-53.

41. Hooper LV, Stappenbeck TS, Hong CV, Gordon Jl. Angiogenins: a new class of microbicidal proteins involved in innate immunity. Nat Immunol. 2003;4: 269-73.

42. Malamitsi-Puchner A, Sarandakou A, Baka S, Hasiakos D, Kouskouni E, Creatsas G. In vitro fertilization: angiogenic, proliferative, and apoptotic factors in the follicular fluid. Ann N Y Acad Sci. 2003:997:124-8.

43. Kawano Y, Zeineh Hasan K, Fukuda J, Mine S, Miyakawa I. Production of vascular endothelial growth factor and angiogenic factor in human follicular fluid. Mol Cell Endocrinol. 2003;202:19-23.

44. Stouffer RL, Martinez-Chequer JC, Molskness TA, Xu F, Hazzard TM. Regulation and action of angiogenic factors in the primate ovary. Arch Med Res. 2001;32:567-75.

45. Malamitsi-Puchner A, Sarandakou A, Baka SG, Tziotis J, Rizos D, Hassiakos D, Creatsas $\mathrm{G}$. Concentrations of angiogenic factors in follicular fluid and oocyte-cumulus complex culture medium from women undergoing in vitro fertilization: association with oocyte maturity and fertilization. Fertil Steril. 2001;76:98-101

46. Israely T, Nevo N, Harmelin A, Neeman M, Tsafriri A. Reducing ischaemic damage in rodent ovarian xenografts transplanted into granulation tissue. Hum Reprod. 2006;21:1368-79.

47. Wu D, Lei Y, Tong Y, Tang F, Qian Y, Zhou Y. Angiogenesis of the frozenthawed human fetal ovarian tissue at the early stage after xenotransplantation and the positive effect of Salviae miltiorrhizae. Anat Rec (Hoboken). 2010;293: 2154-62.

48. Kim SS. Assessment of long term endocrine function after transplantation of frozen-thawed human ovarian tissue to the heterotopic site: 10 year longitudinal follow-up study. J Assist Reprod Genet. 2012;29:489-93.

49. Nugent D, Newton H, Gallivan L, Gosden RG. Protective effect of vitamin E on ischaemia-reperfusion injury in ovarian grafts. J Reprod Fertil. 1998;114: 341-6. 
50. Dath C, Dethy A, Van Langendonckt A, Van Eyck AS, Amorim CA, Luyckx V, Donnez J, Dolmans MM. Endothelial cells are essential for ovarian stromal tissue restructuring after xenotransplantation of isolated ovarian stromal cells. Hum Reprod. 2011:26:1431-9.

51. Poirot C, Abirached F, Prades M, Coussieu C, Bernaudin F, Piver P. Induction of puberty by autograft of cryopreserved ovarian tissue. Lancet. 2012;379: 588.

52. Lee DM, Yeoman RR, Battaglia DE, Stouffer RL, Zelinski-Wooten MB, Fanton JW, Wolf DP. Live birth after ovarian tissue transplant. Nature. 2004;428:137-8

53. Labied S, Delforge Y, Munaut C, Blacher S, Colige A, Delcombel R, Henry L, Fransolet M, Jouan C, Perrier d'Hauterive S, et al. Isoform 111 of vascular endothelial growth factor (VEGF111) improves angiogenesis of ovarian tissue xenotransplantation. Transplantation. 2013;95:426-33.

\section{Submit your next manuscript to BioMed Central} and we will help you at every step:

- We accept pre-submission inquiries

- Our selector tool helps you to find the most relevant journal

- We provide round the clock customer support

- Convenient online submission

- Thorough peer review

- Inclusion in PubMed and all major indexing services

- Maximum visibility for your research

Submit your manuscript at www.biomedcentral.com/submit 